\title{
Synchronization and Clustering in a Multimode Quantum Dot Laser
}

\author{
Yann Tanguy, John Houlihan, and Guillaume Huyet \\ Tyndall National Institute and Physics Department, Lee Maltings, National University of Ireland, University College, Cork, Ireland \\ Evgeny A. Viktorov and Paul Mandel \\ Optique Nonlinéaire Théorique, Université Libre de Bruxelles, Campus Plaine CP 231, B-1050 Bruxelles, Belgium
}

(Received 28 June 2005; published 7 February 2006)

\begin{abstract}
We analyze experimentally the intensity oscillations of the longitudinal modes of quantum dot semiconductor lasers. We show that the modal intensities can oscillate chaotically with different average frequencies, but obey a highly organized antiphase dynamics leading to a constant total output power. The fluctuations are in the $\mathrm{MHz}$ range. We report the first experimental observation of frequency clustering associated with synchronization. We also observe the propagation of perturbations across the optical spectrum from blue to red.
\end{abstract}

DOI: 10.1103/PhysRevLett.96.053902

Coupled oscillators exhibit many interesting features that appear naturally in everyday life [1,2]. Such systems can be broadly divided into either globally coupled, where each oscillator is directly influenced by each of the other oscillators, or locally coupled, where nearest-neighbor interactions dominate. Synchronization occurs in both types of systems. The appearance of one or more clusters, where each cluster consists of a subset of synchronized oscillators, was predicted for globally coupled systems in the framework of the Kuramoto model [3]. In lasers, synchronization has been harnessed to achieve phase locked arrays of lasers which deliver high brightness outputs (local coupling) [4] and mode-locked lasers resulting in very short optical pulses (global coupling). Fundamental studies of chaotic state locking have also been examined in multimode lasers; e.g., two coupled modes of a ring laser can exhibit chaotic phase synchronization [5]. Globally coupled multimode lasers also exhibit antiphase dynamics [6,7]. Antiphase dynamics was studied theoretically and experimentally in diverse globally coupled systems such as Josephson junctions [8], chemical oscillators [9], and olfactory systems [10].

The aim of this Letter is to report the first experimental evidence, to our best knowledge, of clustering effects associated with synchronization. This was achieved by analyzing the intensity oscillations of the longitudinal modes of quantum dot semiconductor lasers. Each laser has up to 40 longitudinal modes where each lasing mode displays large amplitude chaotic fluctuations although the total laser output power remains almost constant. The fluctuations measured in each mode occur at frequencies of tens of $\mathrm{MHz}$, which is much smaller than the frequency difference between two consecutive modes $(25 \mathrm{GHz})$ and the laser's relaxation oscillation frequency $(>1 \mathrm{GHz})$. Modal fluctuations in quantum well lasers at similar frequencies, known as mode partition, have previously been interpreted as a noise induced phenomenon [11]. However, our measurements indicate several deterministic features which cannot be accounted for by such a description. We
PACS numbers: 42.65.Sf, 05.40.Ca, 05.45.-a, 42.65.Pc

observe that groups of modes may have the same frequency indicating the appearance of clustering. We also observe the propagation of perturbations across the optical spectrum from blue to red similar to those observed in quantum well semiconductor lasers [12]. Transient propagation of waves in the spectrum of a laser was previously observed in a fiber laser [13]. In the present case the perturbations consist of chaotic deviations from a mean value as opposed to the situation with quantum well devices where only one mode appears at any time. With increasing injection current, this propagation becomes more regular and results in a constant propagation velocity over the measurement range.

The devices used for this experiment were the same as reported in [14] and were fabricated using InAs quantum dot material emitting at $1310 \mathrm{~nm}$ [15]. The self-organized quantum dot active region heterostructure consisted of six InAs quantum dot layers embedded in quantum well using DWELL technology. The ground state wavelength of such structures was around $1310 \mathrm{~nm}$. Single transverse mode ridge waveguide (3-5 $\mu \mathrm{m}$ wide, $0.9 \mu \mathrm{m}$ deep, $1.5 \mathrm{~mm}$ long) index guided lasers were fabricated and operated at room temperature under dc biasing. The laser operated on many longitudinal modes, from less than 10 to above 40 depending on the pump current.

To analyze the multimode dynamics in these structures, a monochromator was used to separate individual longitudinal modes, whose intensity was coupled to a high bandwidth detector (Newport AD300, rise time 300 ps). As the power spectrum exhibited fluctuations at frequencies lower than $50 \mathrm{MHz}$, we replaced our high speed detector with a lower bandwidth amplified InGaAs detector $(50 \mathrm{MHz}$, Thorlabs PDA255). A typical time trace of an individual mode is shown in Fig. 1. For a fixed current level, each mode displayed strong fluctuations (ac/dc standard deviation around 40\%), while the total power remained nearly flat, demonstrating the appearance of antiphase dynamics. In order to ascertain whether the underlying dynamics is of a stochastic or deterministic nature, we measured the cor- 


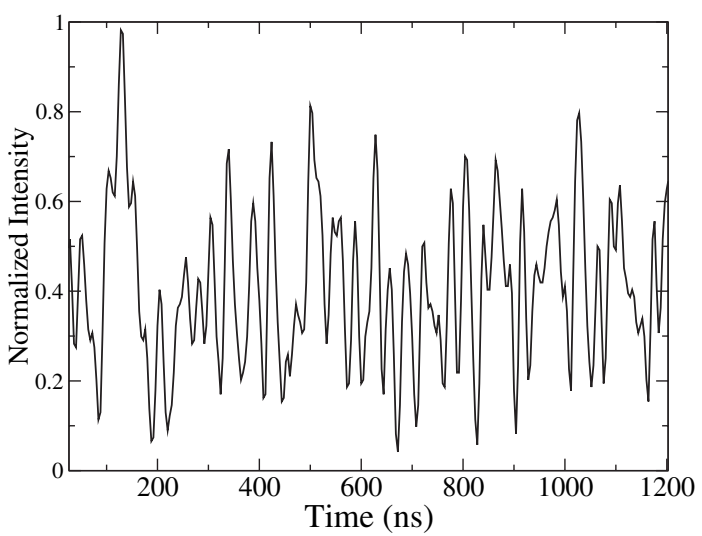

FIG. 1. Example of a chaotic time trace from one longitudinal mode at $3 I_{\text {th }}$ recorded on a digital ocsilloscope.

relation dimension [16] as a means to evaluate the fractal dimension. Usually, low dimensional chaos leads to a fractional value of the correlation dimension between 2 and 3, while high dimensional chaos or noise leads to much larger values. In the case of our experiment, the correlation dimension of the fluctuations in each mode was between 2.8 and 3.3 at $3 I_{\text {th }}\left(I_{\text {th }}=40 \mathrm{~mA}\right)$ depending on the mode number demonstrating the deterministic origin of these fluctuations. Both the number of longitudinal modes and the average frequency of fluctuations in each longitudinal mode increased with injection current. The peak frequency of fluctuations is between 10 and $30 \mathrm{MHz}$ as shown in Fig. 2. The most interesting feature of the observed fluctuations is the difference in the power spectra of individual mode fluctuations. The modes can have either the same or sufficiently different frequencies of fluctuations, forming the groups of modes fluctuating at the same frequency and therefore exhibiting clustering phenomena. It was also noted that the spread of peak frequencies narrows with increasing current from $11 \mathrm{MHz}$ (at $3 I_{\text {th }}$ ) to $3 \mathrm{MHz}$ (at $\left.4 I_{\text {th }}\right)$.

As the phase relations between coupled oscillators usually provide insights about their interaction we measured the phase difference between each mode and some reference mode. For nonperiodic signals, the Hilbert transform is commonly used to extract phase information [17] and
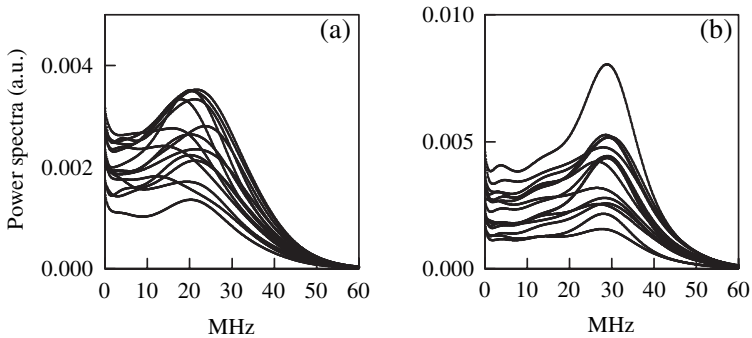

FIG. 2. Power spectra calculated from the Fourier transforms of recorded intensity time traces (over $1 \mathrm{~ms}$ time span) for a representative sample of longitudinal modes at (a) $3 I_{\text {th }}$ (the spread is $11 \mathrm{MHz}$ ) and (b) $4 I_{\text {th }}$ (the spread is $3 \mathrm{MHz}$ ). study phase synchronization of chaotic oscillators [18]. In semiconductor lasers, this technique has been successfully applied to study the effects of external optical feedback [19]. In our experiment, a second detector was used to monitor the output of a reference mode, measured simultaneously with one of the lasing modes. For each pair, the evolution of the Hilbert phase difference with some reference mode, and the normalized cross-correlation function were calculated. The use of a reference mode ensured stable behavior throughout the measurement. This experiment was repeated with different reference modes and the same results were obtained. The typical evolution of the Hilbert phases is shown in Fig. 3(a). We note that some modes have a phase difference with the reference mode which is bounded while others are unbounded. In addition, a locking range can be observed in some pairs of bounded modes indicating the appearance of synchronization as previously described in [18]. For each mode we calculate the average frequency as the average time derivative of the Hilbert phase. In Fig. 3(b), where the average frequency difference with a reference mode is plotted as a function of mode number, the formation of clusters can be seen. We notice that the modes are divided in a subset of clusters, and in each cluster, all the modes have the same frequency. A cluster can be composed of modes from different domains of the optical spectrum. This clustering is observed for a wide parameter range, but the number of clusters and the number of modes within a cluster can vary with pumping current. As the injection current increases, the number of modes increases while the frequency spread decreases indicating a higher degree of synchronization. a)

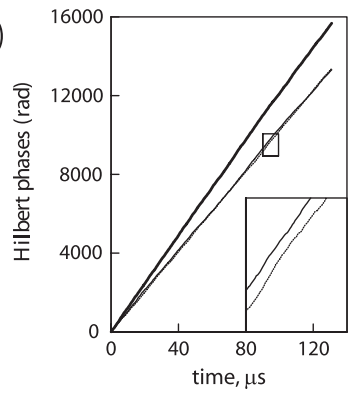

b)

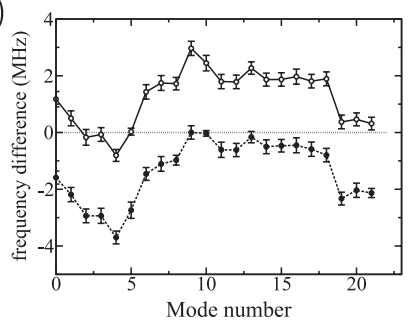

FIG. 3. (a) Hilbert phases for three modes. The reference mode is plotted with a thin solid line. The difference between the phase of the reference mode and the phase of the mode plotted with dotted line is bounded and therefore these modes belong to the same cluster. The difference between the phase of the reference mode and the mode plotted with thick solid line is unbounded. (b) The frequency difference (calculated as the mean of the derivative of the Hilbert phase difference) between the reference mode and each remaining mode in the optical spectrum for two different reference frequencies (mode number 5, open circles; mode number 10 , solid circles). Note that some of the modes form clusters. For example, the sets of modes $(1,19-21)$ and $(6-$ $8,11-12,14-18)$ each form separate clusters. The error bars correspond to the standard deviation of the frequency with time in each case. 
There are too many modes to simultaneously access their time evolution. Therefore, the cross correlation of each mode with the reference mode was calculated from the time series recorded on a digital oscilloscope. In order to assess the degree of correlation, the absolute value of the normalized cross correlation was calculated. This quantity is equal to 1 when the two signals are identical apart from a time shift. If the two modes are independent, then the normalized cross correlation is zero. The degree of correlation between the modes remains low, almost constant across the optical spectrum and increases with increasing injection current from typically 0.1 near threshold to 0.3 at high injection current. Such behavior is consistent with the reduction of spread of frequencies and reflects the increased synchronization of our system. Furthermore, the time lag corresponding to the maximum correlation with the reference mode was calculated for all lasing modes. The results for $3 I_{\text {th }}$ and $4 I_{\text {th }}$ are shown on Fig. 4. At the lower current, the time lag displays an increase in average from blue to red over the spectral range with strong deviations from mode to mode [Fig. 4(a)]. At the higher current, these fluctuations are much reduced and we observe that the time lag for maximum correlation increases linearly from mode to mode as shown on Fig. 4(b). The linear dependence at high currents indicates that the disordered sequence of switching between the modes becomes more regular. This behavior is consistent with the increased synchronization observed in the previous section.

Finally, in order to characterize the chaotic nature of the observed fluctuations, we measured the correlation dimension of the modal signals as already discussed in the introduction. The modes from the central region of the spectrum have lower values of the correlation dimension, $\approx 2.8$, and the modes at the edges have higher values, $\approx 3.3$. The trend is similar to the distribution of frequencies across the spectrum suggesting that the modes from different clusters can exhibit different levels of complexity. The higher correlation dimension corresponds to the modes at the edges of optical spectrum which have stronger influence of noise. We link the difference in the calculated
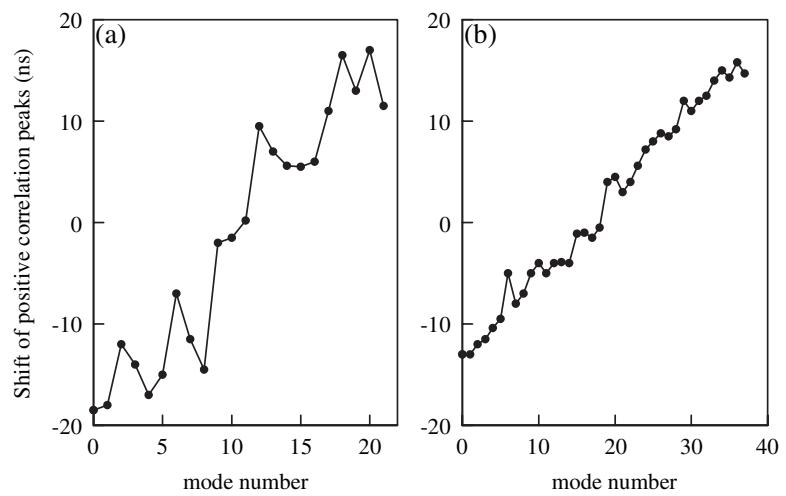

FIG. 4. Time lag corresponding to the maximum of the correlation function with respect to a fixed reference mode, for each longitudinal mode at (a) $3 I_{\text {th }}$ and (b) $4 I_{\text {th }}$. correlation dimensions to the stochastic processes which govern the appearance of new lasing modes at the edges of optical spectrum.

These experimental results provide important insights into the multimode quantum dot laser behavior. As noted in the introduction, the modal fluctuations characterized in this Letter occur in the same frequency range as modal fluctuations observed in quantum well devices and reported as mode partition noise [11]. However, the observation of a low correlation dimension, clustering of the oscillation frequencies and propagation waves in the optical spectrum indicates the presence of a strong underlying deterministic mechanism. Indeed, these experimental results should play an important role in the development of future quantum dot laser models. The essential features that should be included are as follows: (i) Globally coupled longitudinal modes can display antiphase dynamics with nearly complete compensation in the total output; (ii) they can have different average frequencies of fluctuations; (iii) they may form clusters; and (iv) they can exhibit ordered perturbation waves through the modal spectrum.

Previous studies have shown that laser antiphase dynamics of the modal intensities with nearly complete compensation in the total output can be described by a rate equation model for globally coupled lasing modes [2023]. Therefore, we first divide the ensemble of quantum dots into $N$ groups, where $N$ is the number of lasing modes. Each group contains the dots with resonant energy for interband transition corresponding to one of the lasing modes. We may assume that the complex modal fields $E_{j}(t)$ can be described by rate equations which are globally coupled to the integrated populations of the dots within the group $n_{j}(t)$. In its simplest version, the model is

$$
\begin{gathered}
\frac{d E_{j}}{d t}=\frac{1}{2}(1-i \alpha)\left[G_{j}\left(\left\{n_{k}, E_{k} ; k=1, \ldots, N\right\}\right)-1\right] E_{j}, \\
\eta \frac{d n_{j}}{d t}=P\left(1-n_{j}\right)-n_{j}-\sum_{k}^{N} g_{j k}\left(\left\{n_{\ell}, E_{\ell} ; \ell\right.\right. \\
=1, \ldots, N\})\left|E_{k}\right|^{2},
\end{gathered}
$$

where $P$ is the pump normalized to its threshold value, $\eta=$ $\tau_{c} / \tau_{p}$ is the ratio between the carrier lifetime and the cavity round trip time, $\alpha$ is the linewidth enhancement factor, and $1-n_{j}$ is the Pauli blocking factor. The modal gains $G_{j}\left(\left\{n_{k}, E_{k} ; k=1, \ldots, N\right\}\right)$ and the cross-couplings coefficients $g_{j k}\left(\left\{n_{\ell}, E_{\ell} ; \ell=1, \ldots, N\right\}\right)$ typically depend on four-wave mixing processes and inhomogeneous broadening [12,24].

Equations (1) and (2) lead to $N$ relaxation oscillation frequencies. The largest is practically equal to the usual single mode relaxation oscillation frequency while all the others are much smaller and close to each other. The frequency separation among the oscillating modes is extremely small compared with the optical frequencies. Therefore, in a good first approximation, one may assume that all frequency-dependent parameters are equal. This 
leads to a highly degenerate set of equations which requires the use of group theoretical tools [7]. This is how the model was analyzed for solid-state lasers and for bulk and multiple quantum well semiconductor lasers. As such, the model accounts for antiphase dynamics of the modal intensities with nearly complete compensation in the total output. What is particular to quantum dot lasers is that the modes have different average oscillation frequencies. We relate this nondegeneracy to the high degree of inhomogeneous broadening. Considering a weak coupling between the modes, Eqs. (1) and (2) may be reduced to a set of globally coupled phase equations which are known to also display antiphase dynamics [25] and clustering [26].

A critical result for quantum dot laser modeling is the observation of a selective blue-to-red propagation of the perturbations. It is important because the degenerate set of equations derived from Eqs. (1) and (2) predicts $(N-1)$ ! equally probable channels of propagation for the perturbations of $N$ lasing modes [6]. The fact that only one propagation channel is observed implies a spontaneous symmetry breaking. Clearly, this channel selection is not related to the linear gain distribution or other dispersion features neglected to derive the degenerate rate equations. This symmetry breaking was recently reported for multiple quantum well multimode semiconductor lasers [12]. Modeling is more advanced for those lasers and four-wavemixing together with a finite $\alpha$ factor could be unambiguously assigned as the origin of this selective blue-to-red propagation mode. The $\alpha$ factor is a global measure of the phase-amplitude coupling. It characterizes the spectral asymmetry in the multimode regime. Quantum dot lasers have a lower $\alpha$ factor, and each mode has a nearly symmetric spectral profile at low current. This symmetry prevents the selection of a unique sequence of switching. As a result, the distribution of the average phases of the chaotic signal is disordered as demonstrated by the correlation measurements in Fig. 4(a). Higher injection currents lead to an increase in the $\alpha$ factor and the asymmetry of the modal spectral profile, eventually leading to excited state lasing [27]. This asymmetry, together with the increase of the $\alpha$ factor, results in an ordered perturbation wave moving from the bluest to the reddest mode as in quantum well lasers.

In conclusion, we have experimentally demonstrated that for quantum dot semiconductor lasers, the modal output powers display chaotic antiphase oscillations while the total output power remains constant. These chaotic oscillations, which are in the $\mathrm{MHz}$ range and are much lower than any intrinsic frequencies in the device, exhibit frequency clustering and propagate across the optical spectrum.

We gratefully acknowledge Brian Corbett for the fabrication of the devices and Zia Laser for wafer supply. This study was supported in Ireland by the Science Foundation Ireland under Contract No. /01/fi/co13 and the Irish Higher Education Authority under the PRTLI program, and in Brussels by the Fonds National de la Recherche
Scientifique (Belgium) and the Interuniversity Attraction Pole Programme-Belgian Science Policy.

[1] A.S. Pikovsky, M. Rosenblum, and J. Kurths, Synchronization: A Universal Concept in Nonlinear Science (Cambridge University Press, Cambridge, 2001).

[2] S. H. Strogatz, Physica (Amsterdam) 143D, 1 (2000).

[3] Y. Kuramoto, Chemical Oscillations, Waves and Turbulence (Springer, Berlin, 1984).

[4] D. Botez and D. R. Scifres, Diode Laser Arrays (Cambridge University Press, Cambridge, 1994).

[5] D. Y. Tang and N. R. Heckenberg, Phys. Rev. E 55, 6618 (1997).

[6] P. Mandel, Theoretical Problems in Cavity Nonlinear Optics (Cambridge University Press, Cambridge, 2005) (paperback edition).

[7] A. G. Vladimirov, E. A. Viktorov, and P. Mandel, Phys. Rev. E 60, 1616 (1999).

[8] P. Hadley and M. R. Beasley, Appl. Phys. Lett. 50, 621 (1987).

[9] M. Yoshimoto, K. Yoshikawa, Y. Mori, and I. Hanazaki, Chem. Phys. Lett. 189, 18 (1992).

[10] W. J. Freeman and C. A. Skarda, Brain Res. Rev. 10, 147 (1985).

[11] G. Agrawal, Phys. Rev. A 37, 2488 (1988).

[12] A. M. Yacomotti, L. Furfaro, X. Hachair, F. Pedaci, M. Giudici, J. Tredicce, J. Javaloyes, S. Balle, E. A. Viktorov, and P. Mandel, Phys. Rev. A 69, 053816 (2004).

[13] C. Szwaj, S. Bielawski, and D. Derozier, Phys. Rev. Lett. 77, 4540 (1996).

[14] J. Muszalski, J. Houlihan, G. Huyet, and B. Corbet, Electron. Lett. 40, 428 (2004).

[15] T. Newell, D. Bossert, A. Stinz, B. Fuchs, and K. Malloy, IEEE Photonics Technol. Lett. 11, 1527 (1999).

[16] G. Grassberger and I. Procaccia, Physica (Amsterdam) 9D, 189 (1983).

[17] D. Gabor, J. Inst. Electr. Eng. 93, 429 (1946); M. Born and E. Wolf, Principles of Optics (Cambridge University Press, Cambridge, 1999), 7th ed.

[18] M. G. Rosenblum, A.S. Pikovsky, and J. Kurths, Phys. Rev. Lett. 76, 1804 (1996).

[19] W.S. Lam, P.N. Guzdar, and R. Roy, Phys. Rev. E 67, 025604 (2003).

[20] K. Otsuka, M. Georgiou, and P. Mandel, Jpn. J. Appl. Phys. 31, L1250 (1992).

[21] D. Pieroux and P. Mandel, Opt. Commun. 107, 245 (1994).

[22] B. A. Nguyen and P. Mandel, Opt. Commun. 138, 81 (1997).

[23] P. Mandel, E. A. Viktorov, C. Masoller, and M. Torre, Physica (Amsterdam) 327A, 129 (2003).

[24] M. Sugawara, K. Mukai, Y. Nakata, H. Ishikawa, and A. Sakamoto, Phys. Rev. B 61, 7595 (2000).

[25] K. Okuda, Physica (Amsterdam) 63D, 424 (1993).

[26] Y. Maistrenko, O. Popovych, O. Burylko, and P. A. Tass, Phys. Rev. Lett. 93, 084102 (2004).

[27] A. Markus, J. Chen, O. Gauthier-Lafaye, J.-G. Provost, C. Paranthoen, and A. Fiore, IEEE J. Sel. Top. Quantum Electron. 9, 1308 (2003). 